\title{
BARDET-BIEDL SYNDROME
}

\author{
Jayashree S. Shah'1, Satya Swapnika Y2, Shruti Singh ${ }^{3}$
}

1 Professor, Department of Ophthalmology, Sri Siddhartha Medical College, Tumkur. 2Junior Resident, Department of Ophthalmology, Sri Siddhartha Medical College, Tumkur. 3Junior Resident, Department of Ophthalmology, Sri Siddhartha Medical College, Tumkur.

\section{ABSTRACT}

\section{BACKGROUND}

Bardet-Biedl syndrome is extremely rare in India. This case report deals with this rare entity. In our case report, we are describing an 11-year-old girl with cardinal symptoms of Bardet-Biedl syndrome as described in literature like Retinitis Pigmentosa, Obesity, Polydactyly, Brachydactyly and Mental Retardation. A diagnosis of Bardet-Biedl syndrome was suggested by clinical findings and confirmed by genetic study.

\section{KEYWORDS}

Bardet-Biedl Syndrome, Retinitis Pigmentosa.

HOW TO CITE THIS ARTICLE: Shah JS, Swapnika SY, Singh S. Bardet-Biedl syndrome. J. Evolution Med. Dent. Sci. 2016;5(101): 7448-7449, DOI: $10.14260 /$ jemds/2016/1686

\section{BACKGROUND}

Bardet-Biedl Syndrome (BBS) is a rare autosomal recessive disorder. ${ }^{1}$ BBS was first described by Bardet and Biedl in 1920. The principal manifestations are rod-cone dystrophy (Retinitis pigmentosa), postaxial polydactyly, central obesity, mental retardation, hypogonadism and renal dysfunction. ${ }^{2}$ Other features not always present include hepatic fibrosis, diabetes mellitus, neurological, speech and language deficits, behavioural traits, facial dysmorphism, dental anomalies and developmental delay. ${ }^{1,2}$

\section{Pathophysiology of Bardet-Biedl Syndrome}

This syndrome is due to mutations in at least 14 different genes, most commonly called as BBS genes which affect the cilia, important for chemical signalling pathways during development. Disruption of the cilia leads to abnormalities of sensory perception. About $1 / 4^{\text {th }}$ of all cases of BBS result from mutations in the BBS1 gene 3 ; $20 \%$ of cases are because of mutations in the BBS10 gene. $2,3,4$ In about $25 \%$ of people with Bardet-Biedl syndrome, the cause of the disorder is unknown. 5

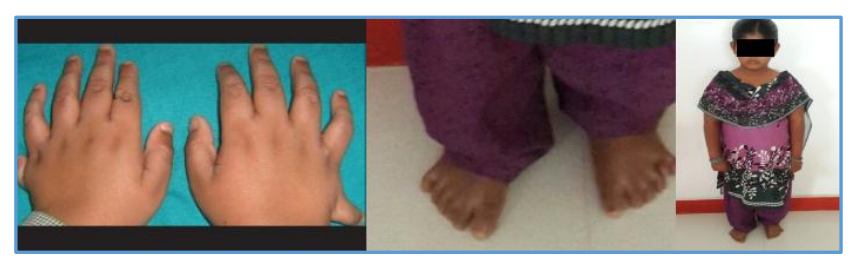

Figure 1

We are presenting here a case of BBS, which is rarely encountered in clinical practise - BBS with a normal genotype.

Financial or Other, Competing Interest: None.

Submission 13-11-2016, Peer Review 07-12-2016,

Acceptance 13-12-2016, Published 19-12-2016.

Corresponding Author:

Dr. Satya Swapnika Y,

Department of Ophthalmology,

Sri Siddhartha Medical College and Hospital,

Agalakote, Tumkur-572107.

E-mail: swapnika.satya@gmail.com

DOI: $10.14260 /$ jemds $/ 2016 / 1686$

\section{(c) (1) $(9)$}

\section{Clinical Summary}

A female child of 11 years old, product of consanguineous marriage came to our ophthalmology OPD with chief complaints of diminution of vision.

\section{On Examination}

Physical and ophthalmic examination and routine investigations were done.

We have found that the child is short for age, have truncal obesity, polydactyly, brachydactyly and poor learning capability.

On ophthalmic examination, we have found that the patient is having a visual acuity of 6/24 in right eye and 6/36 in left eye. Anterior segment examination was normal. Fundus examination revealed pigment clumps in the periphery with attenuated arteries in both eyes. Suggestive of retinitis pigmentosa.

USG Abdomen to rule out internal pathology and genetic analysis to know the gene defect was advised.

USG Abdomen showed simple cortical cysts measuring $2 * 4$ and $2 * 6$ in the left kidney. Both kidneys are normal in size, shape and echo structure.

Uterus - B/L ovaries are normal.

\section{Chromosomal Analysis}

Cultured peripheral blood from this patient revealed a normal female 46, XX chromosome complement. Genetic analysis revealed no gene abnormality.
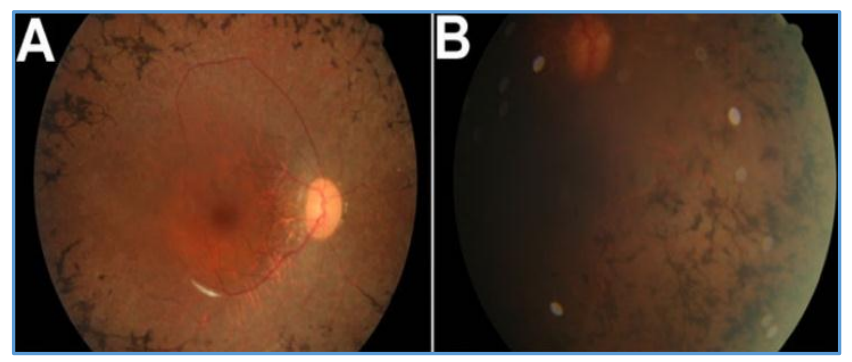

Figure 2. A) Fundus Picture of the Right Eye showing Attenuated Vessels, Tapetal Reflex at the Macula and Pigment Clumps in the Periphery. B) Fundus Picture of the Left Eye showing Peripheral Pigmentary Clumps 


\section{DISCUSSION}

The syndrome was described by Bardet-Biedl in the 1920 . Retinal dystrophy $(100 \%)$ is the $1^{\text {st }}$ major feature of the disorder, $2^{\text {nd }}$ being Obesity $(72-96 \%)$ and $3^{\text {rd }}$ major feature is Limb abnormalities. Obesity usually begins in childhood. Post-axial polydactyly and brachydactyly are most common. Mental retardation is a more disputed feature of BBS. Hypo-genitalism is mostly reported in BBS males than females. In BBS females genital abnormalities include hypoplastic fallopian tubes, uterus and ovaries, partial and complete vaginal atresia, absent vaginal orifice and absent urethral orifice, whereas males have small penis and testes (88\%). Renal failure is the major cause of morbidity and early mortality in BBS. A wide range of renal abnormalities has been described (parenchymal cysts, calyceal clubbing, vesico-ureteric reflux, chronic renal failure, etc.). Mild-to-moderate mental retardation and learning difficulties are additional features of the syndrome.

\section{Differential Diagnosis}

Laurence-Moon syndrome in which retinal pigmentary degeneration, mental retardation and hypogonadism occurs in conjunction with progressive spastic paraparesis and distal muscle weakness.

Alström syndrome is characterised by cone-rod dystrophy, obesity, progressive sensory-neural hearing impairment, dilated cardiomyopathy, the insulin resistance syndrome and developmental delay.

Leber congenital amaurosis, a severe dystrophy of the retina, typically becomes evident in the first year of life.

\section{Management}

Visual rehabilitation is done for the visually impaired. Obesity is managed with diet, exercise and behavioural therapies. Early intervention and special education address cognitive disability. ${ }^{3}$ Renal anomalies and hypertension are treated as in the general population. Hormone replacement therapy for hypogonadism. Surgery to remove accessory digits prevents functional interference and poor fitting of footwear.

\section{CONCLUSION}

When a case of Bardet-Biedl syndrome is suspected, a careful history is taken and a thorough clinical examination is done. Treatment is directed towards specific symptoms and holistic approach is required. Standard care from an Ophthalmologist is in order for the management of refractive error and assistance with low vision aids as vision decreases.

\section{REFERENCES}

1. Kumar S, Mahajan BB, Mittal J, et al. Bardet-Biedl syndrome: a rare case report from North India. Indian J Dermatol Venereol Leprol 2012;78(2):228.

2. Forsythe E, Beales PL. Bardet-Biedl Syndrome. In: Pagon RA, Adam MP, Ardinger HH, et al. eds. Seattle (WA): University of Washington, Seattle 1993-2016.

3. Abu-Safieh L, Al-Anazi S, Al-Abdi L, et al. In search of triallelism in Bardet-Biedl syndrome.Eur J Hum Genet 2012;20(4):420-7.

4. Aldahmesh MA, Li Y, Alhashem A, et al. IFT27, encoding a small GTPase component of IFT particles, is mutated in a consanguineous family with Bardet-Biedl syndrome. Hum Mol Genet 2014;23(12):3307-15.

5. Baker K, Northam GB, Chong WK, et al. Neocortical and hippocampal volume loss in a human ciliopathy: a quantitative MRI study in Bardet-Biedl syndrome. Am J Med Genet A 2011;155A(1):1-8. 